\title{
Experimental Tracking of Limit-Point Bifurcations and Backbone Curves Using Control-Based Continuation
}

\author{
Ludovic Renson*, David A. W. Barton ${ }^{\dagger}$ \\ and Simon A. Neild ${ }^{\ddagger}$ \\ Faculty of Engineering, \\ University of Bristol, Bristol, UK \\ *l.renson@bristol.ac.uk \\ †david.barton@bristol.ac.uk \\ ‡simon.neild@bristol.ac.uk
}

Received August 25, 2016

\begin{abstract}
Control-based continuation ( $\mathrm{CBC}$ ) is a means of applying numerical continuation directly to a physical experiment for bifurcation analysis without the use of a mathematical model. CBC enables the detection and tracking of bifurcations directly, without the need for a post-processing stage as is often the case for more traditional experimental approaches. In this paper, we use CBC to directly locate limit-point bifurcations of a periodically forced oscillator and track them as forcing parameters are varied. Backbone curves, which capture the overall frequency-amplitude dependence of the system's forced response, are also traced out directly. The proposed method is demonstrated on a single-degree-of-freedom mechanical system with a nonlinear stiffness characteristic. Results are presented for two configurations of the nonlinearity - one where it exhibits a hardening stiffness characteristic and one where it exhibits softening-hardening.
\end{abstract}

Keywords: Control-based continuation; experimental bifurcation analysis; bifurcation tracking; backbone curves; softening-hardening nonlinearity.

\section{Introduction}

Experimental data often provides a major contribution to the understanding of complex dynamical systems and allows one to identify the key phenomena and parameters that must be included in a mathematical model to reproduce the system's dominant behaviors (see, for instance, neuron models [Hodgkin \& Huxley, 1952], ocean-atmosphere interactions [Wang \& Picaut, 2013] or even tyre models [Lugner et al., 2005]). Experimental testing is an integral part of the development cycle of most engineering structures and is sometimes a mandatory step for certification (see, aircraft ground vibration testing [Peeters et al., 2008]). Structures are not only tested to make sure they can withstand the external loads they will endure when in operation, but also to extract specific dynamical characteristics key to the development and validation of mathematical models [Ewins, 2000].

Bifurcations represent stability boundaries where dramatic qualitative and quantitative changes in the dynamics of a system can occur

\footnotetext{
*Address for correspondence: University of Bristol, Department of Engineering Mathematics, Merchant Venturers' Building, Woodland Road, BS8 1UB, Bristol, United Kingdom.

This is an Open Access article published by World Scientific Publishing Company. It is distributed under the terms of the Creative Commons Attribution 4.0 (CC-BY) License. Further distribution of this work is permitted, provided the original work is properly cited.
} 
and, as such, they are often key to the understanding of a system's dynamics (see, for instance, the buckling of a structure [Thompson, 2015] or the onset of limit-cycle oscillations in aeroelastic systems [Dimitriadis \& Li, 2009]). Numerical continuation is a popular approach for conducting a bifurcation analysis on a numerical model of a dynamic system. It is a predictor-corrector method that follows paths of steady-state equilibria or periodic response solutions in one or more parameters. One of the benefits of numerical continuation over direct numerical simulations at different parameter values is that the stability and the basins of attraction of the solutions are unimportant to the method, which allows one to track entire solution families and systematically detect bifurcations [Kuznetsov, 2004]. Identified bifurcations can in turn be tracked as parameters are varied. Numerical continuation enables the analysis of a wide class of models, which has led to applications in many different fields, such as in biology with the study of hormone transport models [Draelants et al., 2013], in astrophysics with the study of the three-body problem [Doedel et al., 2003] and in physics with the study of superconductors [Schlomer et al., 2012]. Numerical continuation is also exploited by industry for the design of aircraft components [Sharma et al., 2015]. Numerical continuation software is readily available in the form of AUTO [Doedel et al., 2000], MATCONT [Dhooge et al., 2003] and CoCo [Dankowicz \& Schilder, 2013] amongst others.
Without the need for a mathematical model, control-based continuation $(\mathrm{CBC})$ is a way of applying the concepts behind numerical continuation to a physical system. CBC combines stabilizing feedback control and path-following techniques in order to directly isolate the nonlinear behaviors of interest during experimental tests and track their evolution as parameters are varied. This allows the detection of boundaries between qualitatively different types of behaviors in a robust and systematic way as the experiment is running. This is in direct contrast to standard experimental approaches where any bifurcation analysis must be carried out during a post-processing stage using time series collected at different parameter values [Kerschen et al., 2006].

The concept of CBC was originally presented by Sieber and Krauskopf [2008], and the first experimental demonstration of the method was performed on a parametrically-excited pendulum [Sieber et al., 2010]. Figure 1 illustrates the typical response curves that could be obtained using CBC, considering a Duffing oscillator with cubic nonlinearity as an example. The oscillator's periodic response is studied as a function of two parameters, the frequency and amplitude of the harmonic forcing applied to the system. The solid-black curves obtained at constant forcing amplitudes represent the so-called nonlinear frequency response (NLFR) curves. In practice, the analysis of such curves allows one to detect, for instance, high displacements and stress

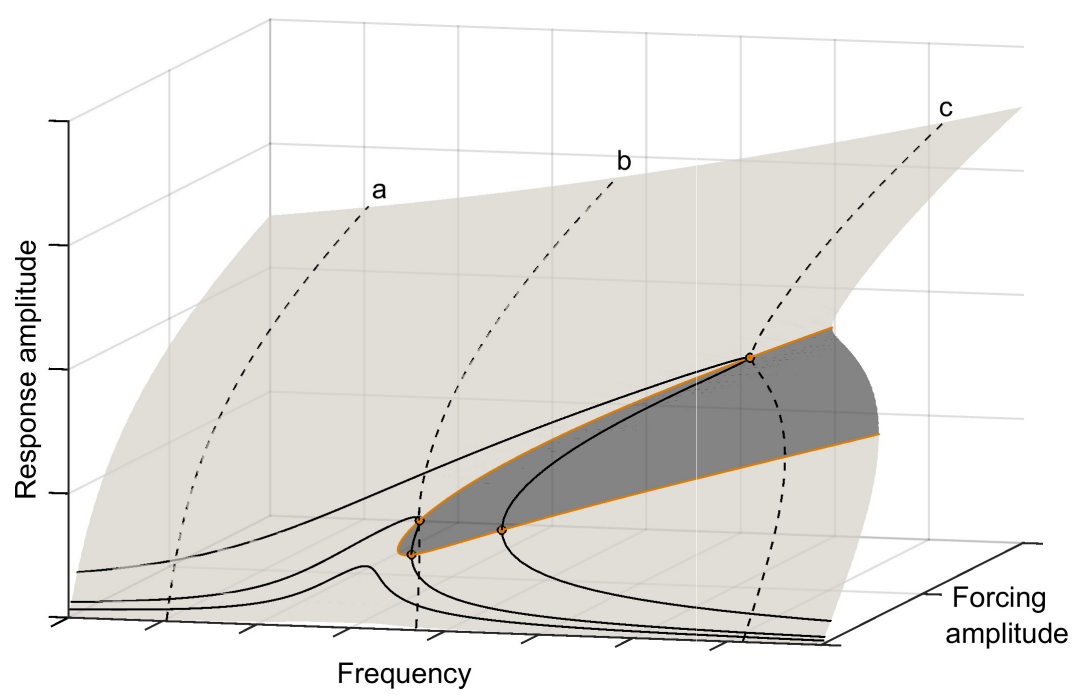

Fig. 1. Typical forced response surface for a Duffing oscillator with darker shading indicating unstable region. The response for constant forcing amplitude and constant forcing frequency are shown by black solid ( -$)$ and dashed (--) lines respectively. Orange dots $(\bullet)$ indicate folds on the constant amplitude curves and orange curve $(-)$ the fold bifurcations. 
levels that can in turn cause failures. The NLFR of a bilinear oscillator and energy harvesters were traced out in the experiment using CBC in [Bureau et al., 2013; Schilder et al., 2015] and [Barton \& Burrow, 2010; Barton \& Sieber, 2013], respectively.

An alternative to NLFR curves is to keep the forcing frequency fixed and consider the response amplitude as the parameter of interest. This results in the curves $(\mathrm{a}-\mathrm{c})$ represented in Fig. 1 by dashed lines. We will refer to these as S-shaped curves to reflect the shape they take when the forcingfrequency is selected such that multiple solutions exist. Compared to NLFR curves, tracing out S-shaped curves does not require sophisticated algorithms because the curve is uniquely parameterized by the response amplitude for any given excitation frequency. This allows us to have simplifications of the path-following techniques used in CBC, resulting in a significant speed up of the method [Barton \& Sieber, 2013].

S-shaped curves can be collected for multiple frequencies to generate a surface mapping the forcing amplitude in function of the forcing frequency and response amplitude (gray surface in Fig. 1). It is possible to exploit this surface to extract relevant dynamic features such as the curve of limit-point (LP) bifurcations (solid orange curve in Fig. 1) [Barton \& Sieber, 2013; Renson et al., 2016b]. LP bifurcation curves contain valuable information about the system's dynamics. For instance, they were used to predict the existence of isolated periodic solutions in the NLFR of various systems [Kuether et al., 2015; Detroux et al., 2015a; Detroux et al., 2015b; Gatti, 2016]. LP bifurcations also represent stability boundaries and mark out the region where hysteretic behavior can be observed when sweeping back and forth the resonance of nonlinear mechanical systems. Note that a LP bifurcation in a NLFR curve is also a LP bifurcation in a S-shape curve as shown in Fig. 1(c).

Using a single-degree-of-freedom nonlinear system with an adjustable softening-hardening restoring force, the first contribution of this paper is to propose a simple method to directly track LP bifurcations in the experiment. The second contribution is to exploit $\mathrm{CBC}$ to measure the backbone curve of the system, that is the response of the unforced, undamped system, further demonstrating the broad applicability of the method presented in [Renson et al., 2016b]. Backbone curves trace out the evolution in frequency of the peak response of the NLFR curves (not represented in Fig. 1 for clarity). In fact, backbone curves govern the evolution of the system's resonance frequencies for increasing vibration amplitudes, which represent a great deal of useful information that can be used to understand the system's dynamics. They can also be exploited to estimate and update model parameters as suggested in [Worden \& Tomlinson, 2001; Dick et al., 2006; Hill et al., 2016; Sracic et al., 2012; Kurt et al., 2015; Peter et al., 2015].

This paper is organized as follows. Section 2 briefly presents CBC and the method used to track backbone curves. A simple algorithm for tracking LP bifurcations is also presented. The set-up used to experimentally demonstrate the methods is detailed in Sec. 3. Two different configurations for the nonlinearity, one exhibiting stiffening and the other softening-stiffening behavior, are considered and the corresponding experimental results are discussed in Secs. 4 and 5, respectively. Finally, Sec. 6 presents the conclusions of this study.

\section{Control-Based Continuation}

At a basic level, numerical continuation tracks the solutions of a zero problem given by

$$
f(x, \lambda)=0
$$

where $x \in \mathbb{R}^{c}$ are the system states and $\lambda \in \mathbb{R}^{d}$ are the system parameters. This allows equilibria and periodic solutions to be found and tracked as the system parameters vary [Seydel, 2010]. To apply a similar idea to an experiment, there are two key challenges to overcome. (1) In general, it is not normally possible to set all the states $x$ of the physical system and so it is not possible to evaluate $f$ at arbitrary points. (2) The physical system must remain around a stable operating point while the experiment is running. While a numerical model going unstable is a mild annoyance, a physical system going unstable can prove dangerous.

In order to address these challenges, a feedback controller is used to stabilize the system and the control target (or reference signal) acts as a proxy for the system state. The feedback control signal takes the general form

$$
u(t)=g\left(x^{*}(t)-x(t)\right)
$$

where $x^{*}(t)$ is the control target, $x(t)$ is the measured (or estimated) state for $x$ and $g$ is a suitable control law. The challenge here is to make the controller noninvasive such that the position 
in parameter space of any invariant sets such as equilibria and periodic orbits is not affected by the controller and is identical to the uncontrolled system of interest. This requirement for noninvasiveness defines the zero problem used in the experiment; a control target must be chosen such that the control action

$$
u(t) \equiv 0 .
$$

Although the control action is equivalently zero, the controller changes the linearization of the dynamics and keeps the whole experiment stable. We note that stability is not sufficient to make an invariant set experimentally observable with reasonable probability and its basin of attraction also has to be large and dense as defined by [Lenci et al., 2013]. However, CBC side steps this issue using continuation (path-following) techniques and can, a priori, reach solutions that have small, eroded basins of attraction. A method of working out the original stability properties of the underlying uncontrolled system without turning off the controller was presented by Barton [2016].

The "full" CBC method combines Newtonlike algorithms with pseudo-arclength continuation techniques to track solutions of (3) and is presented in, for example, [Schilder et al., 2015]. For many forced systems, it is possible to exploit a unique amplitude parameterization of the response (at constant frequency) to derive a simplified CBC method dispensed with derivative calculations and pathfollowing techniques [Barton \& Sieber, 2013]. Before discussing how this simplified CBC method can be used to track backbone (Sec. 2.2) and LP curves (Sec. 2.3), the approach is briefly discussed in the context of capturing S-curves (Sec. 2.1).

Throughout the paper, the dynamics of interest are the periodic responses of a single-degree-offreedom system subject to a single-point, singleharmonic forcing of arbitrary phase $i(t)=$ $a \cos (\omega t)+b \sin (\omega t)$. With this excitation, the state $x(t)$ and control target $x^{*}(t)$ signals can be decomposed into $m$ (finite) Fourier modes

$$
x(t)=\frac{A_{0}}{2}+\sum_{j=1}^{m} A_{j} \cos (j \omega t)+B_{j} \sin (j \omega t)
$$

and

$$
x^{*}(t)=\frac{A_{0}^{*}}{2}+\sum_{j=1}^{m} A_{j}^{*} \cos (j \omega t)+B_{j}^{*} \sin (j \omega t) .
$$

Using Eq. (2) it follows that the control signal may be written in the same form

$$
u(t)=\frac{A_{0}^{u}}{2}+\sum_{j=1}^{m} A_{j}^{u} \cos (j \omega t)+B_{j}^{u} \sin (j \omega t) .
$$

Here, the control signal is applied through the same exciter as the forcing $i(t)$ (i.e. an electrodynamic shaker, see Sec. 3), hence the control signal is superimposed to the excitation and the total external input to the system is given by $r(t)=$ $i(t)+u(t)$, or

$$
\begin{aligned}
r(t)= & \frac{A_{0}^{u}}{2}+\left(a+A_{1}^{u}\right) \cos (\omega t)+\left(b+B_{1}^{u}\right) \sin (\omega t) \\
& +\sum_{j=2}^{m} A_{j}^{u} \cos (j \omega t)+B_{j}^{u} \sin (j \omega t) .
\end{aligned}
$$

Observing the form of this excitation, a significant simplification to the approach may be made, this is to treat the fundamental components of the control signal as part of a redefined excitation $p(t)=$ $i(t)+A_{1}^{u} \cos (\omega t)+B_{1}^{u} \sin (\omega t)=\left(a+A_{1}^{u}\right) \cos (\omega t)+$ $\left(b+B_{1}^{u}\right) \sin (\omega t)$. Using the control target, Eq. (3), may be modified to

$$
\begin{aligned}
& \tilde{u}(t) \equiv 0, \quad \text { where } \\
& \tilde{u}(t)=\frac{A_{0}^{u}}{2}+\sum_{j=2}^{m} A_{j}^{u} \cos (j \omega t)+B_{j}^{u} \sin (j \omega t) .
\end{aligned}
$$

This control target is easier to achieve [Barton \& Sieber, 2013], however the cost is that now the input excitation is no longer fully determined by the user as it contains a component of $u(t)$. In practice, the continuation normally starts at low amplitude so it is convenient to set $a$ and $b$ to zero and let this redefined excitation be purely governed by the fundamental harmonics of the control signal

$$
p(t)=A_{1}^{u} \cos (j \omega t)+B_{1}^{u} \sin (j \omega t) .
$$

The approach for finding a suitable target $x^{*}$ such that Eq. (7) is satisfied is now discussed for the case where the continuation is conducted in amplitude of response.

\subsection{Continuation in amplitude}

The S-curves map the amplitude of the excitation as a function of the system's response amplitude at a fixed frequency. To trace out S-curves, the 
simplified method presented in [Barton \& Sieber, 2013] proceeds in two steps. First, one of the fundamental target coefficients is incremented. Second, the target coefficients are iteratively modified to reduce the control action such that Eq. (7) is satisfied (up to experimental accuracy), at which point the observed dynamics can be recorded.

\section{Algorithm I}

Step I. One of the fundamental target coefficients $A_{1}^{*}$ (or $B_{1}^{*}$ ) is incremented. As a result of a new (nonzero) target coefficient, the controller will act in an attempt to minimize the difference between the response $x(t)$ and the target $x^{*}(t)$. Once the system has reached its steady-state, an approximation to the effective excitation can be found using Eq. (8) (assuming $a=b=0$ ). However, it is unlikely that this is a valid single-frequency forcing solution where Eq. (7) is satisfied because $u(t)$ is likely to contain harmonics due to harmonics in $x(t)$. This is addressed in the next step by modifying the target.

Step II. It is now necessary to recover pure harmonic forcing by finding control target coefficients $\left(A_{0}^{*}, A_{j}^{*}, B_{j}^{*}\right)_{j=2}^{m}$ for which $\left(A_{0}^{u}, A_{j}^{u}, B_{j}^{u}\right)_{(j=2)}^{m}$ are equal to zero, i.e. Eq. (7) is satisfied. This problem represents a nonlinear system of $2 m-1$ equations and $2 m-1$ unknowns. It can be solved using Newton-like methods as in the "full" CBC method (the Jacobian matrix can be computed experimentally using finite differences), or more simply, using a fixed-point iteration algorithm [Barton \& Sieber, 2013].

After convergence, the higher-harmonic coefficients of the control signal equal zero (up to experimental accuracy) and the fundamental coefficients $\left(A_{1}^{u}, B_{1}^{u}\right)$ represent the total forcing applied to the system. At this point, we can claim that the controller is noninvasive and that the position in parameter space of the observed periodic response is identical to the one in the underlying uncontrolled system with an excitation $p(t)=$ $A_{1}^{u} \cos (j \omega t)+B_{1}^{u} \sin (j \omega t)$, see Eq. (8). Note that the controller is still active and maintains the system response at the prescribed target coefficients where the nonfundamental components of the control signal, $\tilde{u}(t)$, are zero, thus avoiding the development of any instabilities. As such, CBC is generally robust to bifurcations and the associated stability changes.
Iterating between these steps with modifications to the target coefficients allow the complete S-curve to be generated.

\subsection{Tracking backbone curves}

The concept of nonlinear normal modes (NNMs) was pioneered in the 1960's by Rosenberg [1960] as a direct conceptual extension of linear normal modes to nonlinear systems. When defined as periodic solutions of the conservative (i.e. undamped, unforced) equations of motions, NNMs form families of periodic solutions that represent the evolution of the resonance frequencies of the system for increasing energy. NNMs have proved useful for analyzing a number of dynamic features of nonlinear systems, including modal interactions [Renson et al., 2015], mode bifurcations [Cammarano et al., 2014], and localization [Vakakis et al., 2008].

NNMs are also useful in the context of damped, forced systems and were used, for instance, to predict the presence of isolated solutions in the NLFR of different systems [Kuether et al., 2015; Hill et al., 2016]. NNMs are often called backbone curves when compared to NLFR curves because they capture the evolution of the resonance peaks in the forced response of the system. Peeters et al. [2011a] have shown that a forced damped system can oscillate according to the NNMs of its underlying conservative system provided that a multiharmonic excitation in phase quadrature with the response is applied to all the degrees of freedom of the system. In fact, the appropriate force distribution (both spatially and harmonically) counterbalances the damping in the structure and allows the isolation of a specific NNM. In the particular case of a single-degree-of-freedom system (as is the case in this paper), several studies have shown that much simpler excitations, single-harmonic and single point, can isolate the NNM of the system [Peeters et al., 2011b; Zapico-Valle et al., 2013].

At a NNM, the phase condition between the harmonic excitation $p(t)$ and the fundamental harmonic component of the system response $x(t)$ can be expressed as a scalar equation

$$
q(\omega)=\left(\phi_{x, 1}(\omega)-\phi_{p, 1}(\omega)\right)+\frac{\pi}{2}=0,
$$

where $\phi_{x, 1}(\omega)$ and $\phi_{p, 1}(\omega)$ are the phases of the fundamental Fourier modes of the response and total input, respectively. 
A point on the backbone curve is obtained when the quadrature condition (9) is satisfied together with the higher-harmonics present in the control signal [see Eq. (7)]. For constant fundamental target coefficients $\left(A_{1}^{*}, B_{1}^{*}\right)$, this condition can be resolved using a Newton-like method, or more simply, a bisection method. The backbone curve is followed for increasing vibration amplitudes by stepping one of the fundamental target coefficients (as in Sec. 2.1). Additional details about the method can be found in [Renson et al., 2016b].

\subsection{Tracking limit-point bifurcations}

Based on the simplified CBC method described above, a simple algorithm to track LP bifurcations is proposed. The authors believe this is the first reporting of a method that can follow such bifurcations directly during experimental tests.

LP bifurcations correspond to specific points in parameter space where two branches of periodic oscillations join together. At the bifurcation point, the response curve has an extremum with respect to the bifurcation parameter [Kuznetsov, 2004]. Although this condition (or similar) can be easily used in numerical simulations to detect and track LP bifurcations, in an experimental context, where noise affects measurements and corrupts derivative calculations, it would be hard to exploit.

The philosophy of the proposed method is markedly different from the approaches used in a numerical context. Realizing that the presence of noise will, in general, prevent any attempt to directly measure a bifurcation point, the method relies on the geometric nature of LP bifurcations (extremum in the bifurcation parameter) to collect suitably positioned data points and estimate the actual position of the bifurcation using a polynomial regression. This approach has the advantage of being simple and also informative as the collected data will convey information about the sharpness of the solution curve around the bifurcation. Moreover, it is more robust to noise as the estimated location of the bifurcation point is based on a series of measurements instead of a single derivative. If necessary, the data points can also be exploited to compute confidence bounds on the identified bifurcation point. This is however not the objective of the paper.

Figure 2 shows the general structure of the algorithm which is divided into five main steps. Step 0 is the initialization of the algorithm. Steps I-III aim to estimate a LP bifurcation point at a fixed forcing frequency, while Step IV is used to predict the position of a new LP bifurcation based on the bifurcation points previously identified. Steps I-IV are first described considering the identification of the $k$ th bifurcation point. The initialization of the algorithm in Step 0 is described afterwards.

\section{Algorithm II}

Step I. The principle of this step is similar to the generation of a S-curve at a fixed forcing frequency. Here, $n$ data points, each with a fundamental target coefficient $A_{1}^{*}$ (or $B_{1}^{*}$ ), that are equally distributed around a predicted bifurcation point $\left(\bar{A}_{1}^{*}, \bar{\omega}, \bar{p}\right)^{k}$ are found. For each target value $A_{1, j}^{*}$, $j=1, \ldots, n$, the applied excitation is estimated using (8) (or directly measured) after canceling the higher-harmonics present in the control signal (cf. Sec. 2.1).

Step II. A polynomial regression is built based on the $n$ data points $\left(A_{1, j}^{*}, p_{j}\right)_{j=1}^{n}$ collected in Step I. The fundamental target coefficient is considered as the independent variable. The position $\left(A_{1}^{*}, p\right)^{k}$ of the LP bifurcation point is then estimated as one of the roots of the polynomial's first-order derivative. In practice, a cubic polynomial is chosen, which has the advantage of allowing the detection of two LP bifurcations within the data, as it can be the

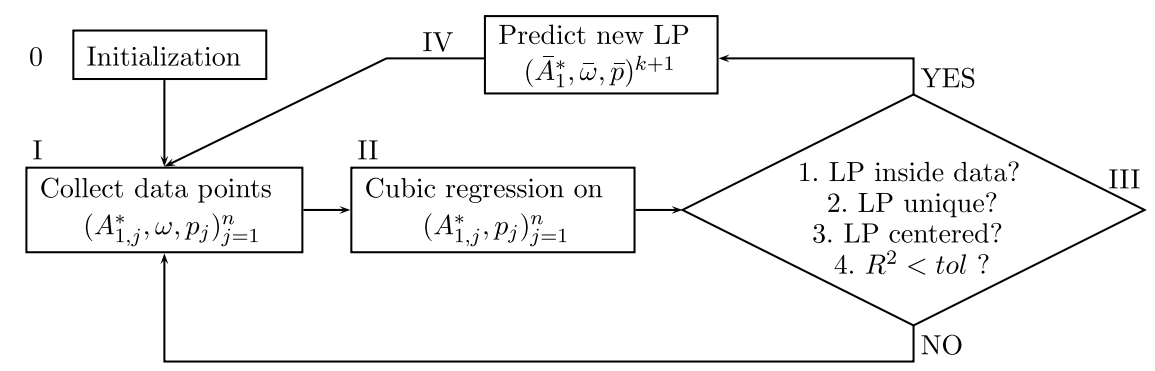

Fig. 2. Block diagram of Algorithm II for tracking LP bifurcations. 


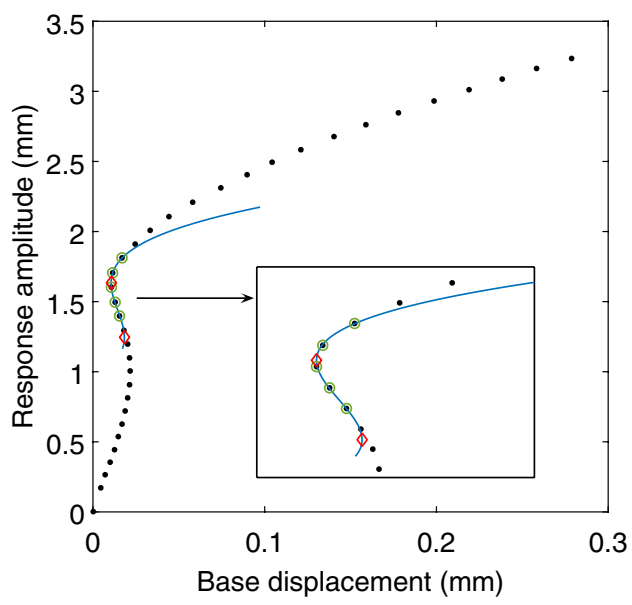

(a)

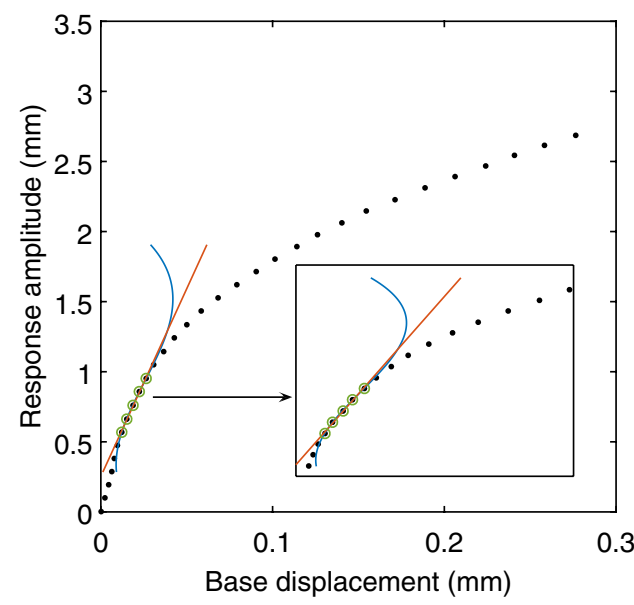

(b)

Fig. 3. Illustration of the polynomial regression applied to two S-curves from Sec. 4. (•) Data point; (०) data used for regression; (-) cubic regression; (-) linear regression; $(\diamond)$ estimated LP bifurcation point.

case when another LP bifurcation curve is crossed (for instance, close to a codimension-two cusp bifurcation).

Figure 3(a) illustrates Step II using experimental data collected in the first nonlinearity configuration (see Sec. 4). From the full S-curve, only five data points, circled in green (o), are considered for the regression. The obtained polynomial fit is represented in blue $(-)$ together with the two real roots of its first-order derivative $(\diamond)$. The root found within the range of the data is considered as the LP of interest.

Step III. The need for additional data is assessed based on the identified LP and the quality of the cubic fit. If two LP bifurcations are detected with the data, the bifurcation point that preserves the curvature, i.e. the orientation of the bifurcation, is kept. Additional data is also collected around the second bifurcation point in order to (1) confirm the finding and (2) further explore the system response. In the particular case where no LP bifurcation is found either because all the roots of the first-order derivative are complex or because they both lie outside the collected data points, additional data is collected for a larger range of $A_{1}^{*}$ (or $B_{1}^{*}$ ) coefficients around the predicted bifurcation point $\left(\bar{A}_{1}^{*}, \bar{\omega}, \bar{p}\right)^{k}$. In this process, the data is also fitted with a linear polynomial and a comparison between the linear and cubic models is performed using leave-one-out cross validation (LOOCV) [Seber \& Lee, 2003]. This criterion represents the average error made in the prediction of a data point when left outside the training data set (i.e. the set of data used to build the model). When the criterion for the cubic model is larger than the criterion for the linear model, the linear model is considered to be more appropriate to represent the data. In this case, it is assumed that the bifurcation curve no longer exists and the algorithm is stopped. This case is illustrated in Fig. 3(b).

When a single LP bifurcation has been identified, the algorithm verifies that the bifurcation does not depend on a single data point and that it is appropriately centered amongst the data points. If this is not the case, the algorithm generates additional data and the polynomial regression step is repeated. The goodness of fit is also checked using the $R^{2}$, or coefficient of determination, criterion [Seber \& Lee, 2003], where $0 \leq R^{2} \leq 1$ with $R^{2}=1$ represents a perfect fit. Additional data is generated if $R^{2}$ is lower than a user defined tolerance tol.

Step $\boldsymbol{I} \boldsymbol{V}$. A new predicted position $\left(\bar{A}_{1}^{*}, \bar{\omega}, \bar{p}\right)^{k+1}$ is generated from the position of the previous two bifurcation points $\left(A_{1}^{*}, \omega, p\right)^{k}$ and $\left(A_{1}^{*}, \omega, p\right)^{k-1}$ using linear extrapolation. A fixed frequency step $\Delta \omega$ is considered such that the prediction is given by

$$
\begin{aligned}
\bar{A}_{1}^{*, k+1} & =A_{1}^{*, k}+\frac{A_{1}^{*, k}-A_{1}^{*, k-1}}{\omega^{k}-\omega^{k-1}} \Delta \omega, \\
\bar{\omega}^{k+1} & =\omega^{k}+\Delta \omega \\
\bar{p}^{k+1} & =p^{k}+\frac{p^{k}-p^{k-1}}{\omega^{k}-\omega^{k-1}} \Delta \omega .
\end{aligned}
$$


The predicted values of the target coefficient and forcing frequency are set to the experimental system and Step I is repeated around the new operating point. The value predicted for the amplitude of excitation is not exploited as it corresponds to a parameter that cannot be set and has to be measured [or estimated using (8)].

Step 0. The experiment is started around a first bifurcation point which can be easily determined using, for instance, the data from a S-curve [as in Fig. 3(a)]. Considering a small frequency step $\Delta \omega_{0}<\Delta \omega$, the position of a second bifurcation point is predicted as being equal to the first bifurcation point: $\left(\bar{A}_{1}^{*, 2}, \bar{\omega}^{2}, \bar{p}^{2}\right)=\left(A_{1}^{*, 1}, \omega^{1}+\Delta \omega_{0}, p^{1}\right)$. The algorithm can then be started, beginning with Step I to find the actual position of the second bifurcation point.

One should note that the control target $A_{1}^{*}$ (and $\left.B_{1}^{*}\right)$ is a proxy for the system's response amplitude. During post-processing, the control target can be replaced by the actual response amplitude and the bifurcation curve represented in the classical (forcing frequency, forcing amplitude, response amplitude) space.

The proposed algorithm monotonously steps along the LP curve in frequency, finding the LP point for each frequency in turn. As such, the algorithm cannot follow the LP bifurcation curve when it doubles back on itself at a cusp bifurcation (see the orange curve in Fig. 1). To address this issue, higher-order prediction strategies that allow to progress along the bifurcation curve even when it changes direction were tested. Although the algorithm successfully passed through the cusp, the method is sensitive to noise. This resulted in a lack of robustness which was deemed not suitable for experiments. Similar conclusions about higherorder prediction methods were found by Schilder et al. [2015].

\section{Experimental Set-Up}

To demonstrate the methods from Sec. 2 the system shown in Fig. 4(a) is considered. This system is made of a thin steel plate clamped to an aluminum armature at one end. At the other end of the plate, two sets of neodymium magnets are attached. The system acts as a single-degree-offreedom (SDOF) oscillator and is fixed vertically to avoid gravity-induced deformations transverse to the plate thickness. Under base excitation, the moving magnets interact with an iron laminated stator and a coil. The magnetic interactions introduce a complex nonlinear restoring force leading to a system whose frequency-amplitude characteristic is first softening and then hardening. The nonlinearity can be adjusted by changing the air gap between the magnets and the iron stator such that the relative importance of the softening region over the hardening region increases for smaller gaps. The system is bistable for very small gaps, but this configuration is not investigated in this paper. The damping in the system can also be adjusted with the load connected to the coil. Here, the circuit is left open producing the smallest possible damping.

As shown in Fig. 4(b), absolute base and plate tip displacements are measured using two Omron

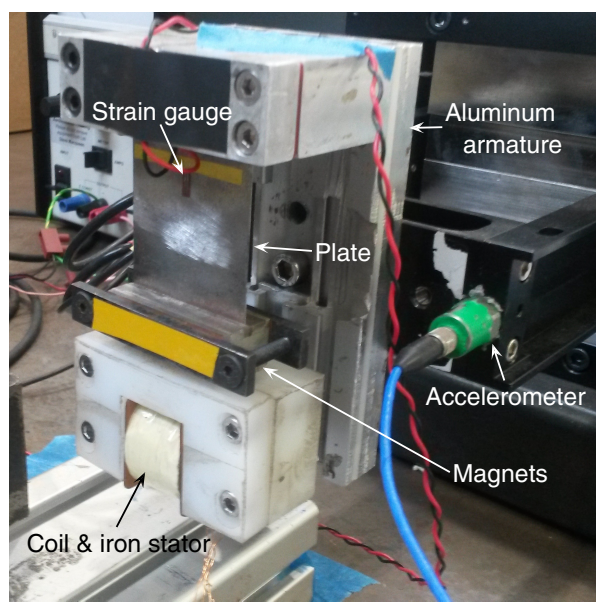

(a)

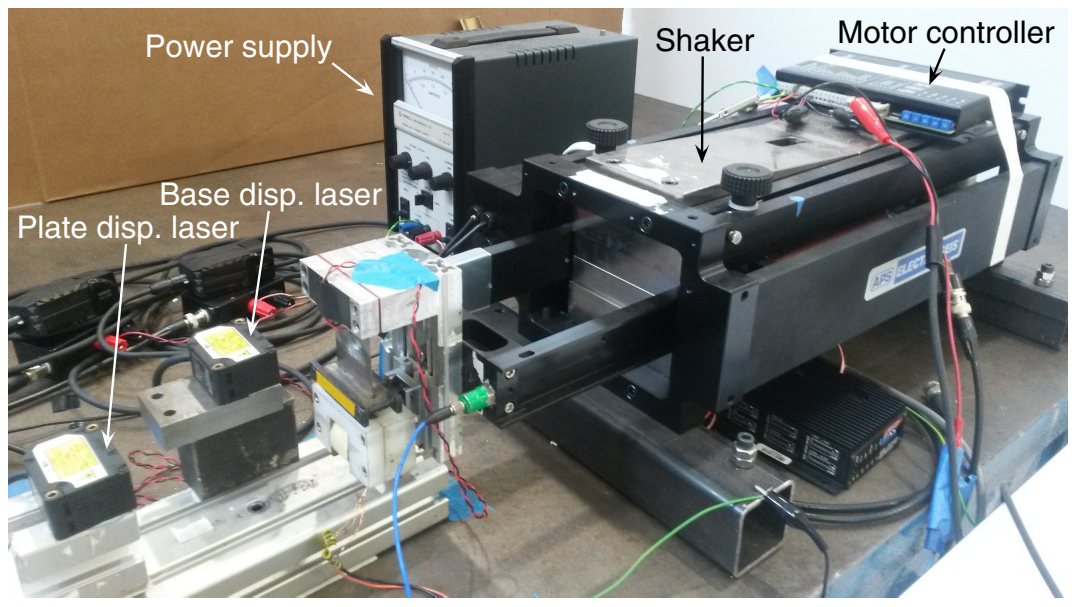

(b)

Fig. 4. (a) Picture of the nonlinear oscillator and (b) picture of the experimental set-up. 
lasers, ZX2-LD50 and ZX2-LD100, respectively. Their sampling period is set to $60 \mu \mathrm{s}$. An accelerometer is attached to the shaker's arm in order to measure base accelerations. A strain gauge also measures the plate deformation at the clamp [Fig. 4(a)].

The nonlinear oscillator is excited at the base by a long-stroke electrodynamic shaker, model APS 113, equipped with linear bearings and operated in current control mode using a Maxon ADS-50/104QDC motor controller. A PID feedback control system is used to center the position of the shaker's arm. Proportional, derivative and integral gains are $0.09,0.0085$, and 0.0080 , respectively. The fine tuning of the control gains was not necessary for CBC to work. A second-order IIR Butterworth filter with a cutoff frequency at $500 \mathrm{~Hz}$ is also applied to the error signal. Note that the amplifier-shaker system was tested independently and was found to introduce small-amplitude higher harmonics even if the original input to the shaker was harmonic. Throughout our experiments, these harmonics were compensated for using the method described by Renson et al. [2016a].

For simplicity, the feedback controller used in CBC, Eq. (2), takes the form of a proportional-plusderivative $(\mathrm{PD})$ control law, such that

$$
u(t)=k_{p}\left(x^{*}(t)-x(t)\right)+k_{d}\left(\dot{x}^{*}(t)-\dot{x}(t)\right) .
$$

The proportional and derivative gains depend on the air gap configuration and will be given in Secs. 4 and 5. The error signal is based on the strain gauge measurement. The latter presents a low level of noise such that the error signal is filtered for frequencies above $2000 \mathrm{~Hz}$.

The controllers for the shaker and CBC are run in parallel. They are implemented on a BeagleBone Black fitted with a custom data acquisition board (hardware schematics and associated software are open source and freely available [Barton, 2015]). All measurements are made at $5 \mathrm{kHz}$ with no filtering. Estimations of the Fourier coefficients of the response, base displacement, and control action are calculated in real time on the control board. However, this was for convenience rather than a necessity.

\section{Large-Gap Configuration}

The distance between the mass and the iron stator is set to approximately $5.7 \mathrm{~mm}$, which is considered as being large. In this configuration, the system presents a very small softening frequency-amplitude characteristic such that the system is essentially hardening. Periodic oscillations are controlled using a PD feedback loop with proportional and derivative gains equal to 0.065 and 0.005 , respectively.

Continuations in amplitude for fixed values of the forcing frequency were carried out between $23.7 \mathrm{~Hz}$ and $25.4 \mathrm{~Hz}$ in steps of $0.1 \mathrm{~Hz}$ using Algorithm I. In total, 780 data points were collected over a total experimental time of approximately $129 \mathrm{~min}$. Recorded Fourier coefficients were averaged over 10 samples. Each modification of the target coefficients $\left(A_{j}^{*}, B_{j}^{*}\right)$ was followed by a maximum wait of $4 \mathrm{sec}$ for the transients to die out, checking every $0.4 \mathrm{sec}$ to see if the Fourier coefficients were stationary. Fourier coefficients were assumed stationary if their absolute and relative variance was lower than $5 \times 10^{-4}$ and $1 \times 10^{-7}$, respectively. Starting from rest, the target coefficient $A_{1}^{*}$ was incremented by steps of $0.05 \mathrm{~mm}$. To reduce the invasiveness of the controller, the maximum difference allowed between the response and target coefficients was set to $5 \times 10^{-4} \mathrm{~mm}$ for each Fourier coefficient. A maximum of 25 fixed-point iterations were allowed to reach convergence.

At each data point, full time series measurements were made. These are shown as black dots in Fig. 5 where the forcing frequency and forcing amplitude (in $\mathrm{mm}$ ) are plotted against the response amplitude. To aid visualization, a continuous surface constructed from the individual data points is plotted in gray. This surface was created using Gaussian Process (GP) regression [Rasmussen \& Williams, 2006]. The popular square exponential kernel is chosen for the covariance function. The hyper-parameters for the Gaussian process are calculated by maximizing their marginal likelihood. The GP regressor maps the excitation amplitude as a function of the excitation frequency and response amplitude, providing a geometrical model of the system's response surface and thus playing the role of surrogate model. This allows us to use numerical continuation techniques to extract geometrical features of the forced-response surface such as the curve of LP bifurcations represented by a thick black line in Fig. 5 and used as a comparative for our direct algorithm. The dark-gray region defined by this curve is a region where periodic solutions of the uncontrolled system are unstable. It would typically be impossible to observe the data points from this region without control. 


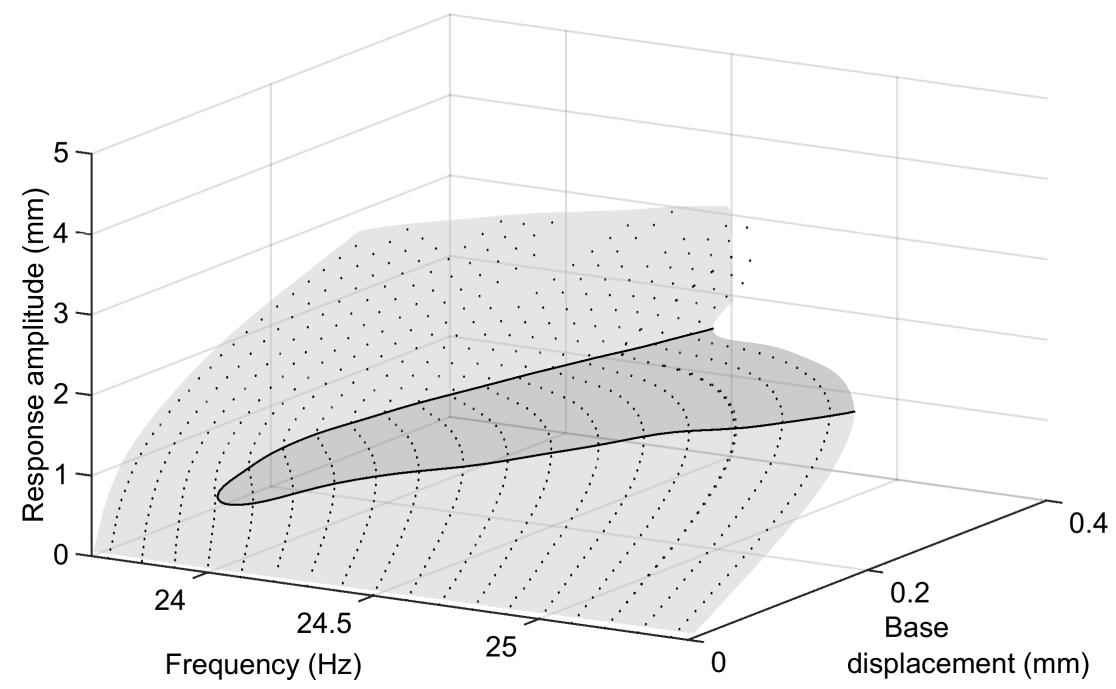

Fig. 5. Forced response of the SDOF shown in Fig. 4. (•) Amplitude of steady-state periodic responses measured during a series of continuations in amplitude (S-curves). (Gray surface) The complete forced-response surface obtained using GP regression. (Dark-gray region) Region where periodic responses are unstable without control. (-) Limit-point bifurcation curve obtained from the GP regression using numerical continuation.

The backbone curve of the system was measured directly by tracking the phase quadrature condition between the system response and the excitation, as explained in Sec. 2.2. Two backbone curves were measured consecutively using the same CBC parameters, but with different initial amplitudes for the continuation. The results are reported in solid blue (-) and dashed orange (--) in Fig. 6 . There is an excellent agreement between the two curves, although slightly larger discrepancies are noticeable between $0.75 \mathrm{~mm}$ and $2 \mathrm{~mm}$. The results show the presence of a softening behavior of approximately $0.1 \mathrm{~Hz}$ for amplitudes lower than $0.4 \mathrm{~mm}$ and a hardening behavior of $1.6 \mathrm{~Hz}$ for amplitudes ranging from $0.4 \mathrm{~mm}$ to $4 \mathrm{~mm}$. Figure 6 also compares the backbone curves with the system NLFR extracted at several constant forcing amplitudes from the GP surrogate model $(-)$. Note that these frequency response curves are not needed for the generation of the backbone curves. They are generated separately for validation purposes. The backbone curve matches the resonance of the system in both the softening and hardening regions very well. This shows excellent consistency between the data sets coming from the S-curves and those coming from the backbone curve tracking.

Starting from the bifurcations detected in the S-curve at $25.4 \mathrm{~Hz}$, the Algorithm II described in Sec. 2.3 was used to track LP bifurcations directly in the experiment. The forcing frequency was first decreased by a frequency step $\Delta \omega_{0}$ of $0.05 \mathrm{~Hz}$ and then by constant steps of $0.1 \mathrm{~Hz}$. Figure 7 compares the measured curves with the curve extracted from the GP regression. Figures 7(b) and 7(c) show two different two-dimensional projections of the surface presented in Fig. 7(a). As explained in Sec. 2.3, the proposed algorithm cannot pass a cusp bifurcation where the LP curve doubles back in frequency (here located around $24 \mathrm{~Hz}$ ). As such, two separate curves

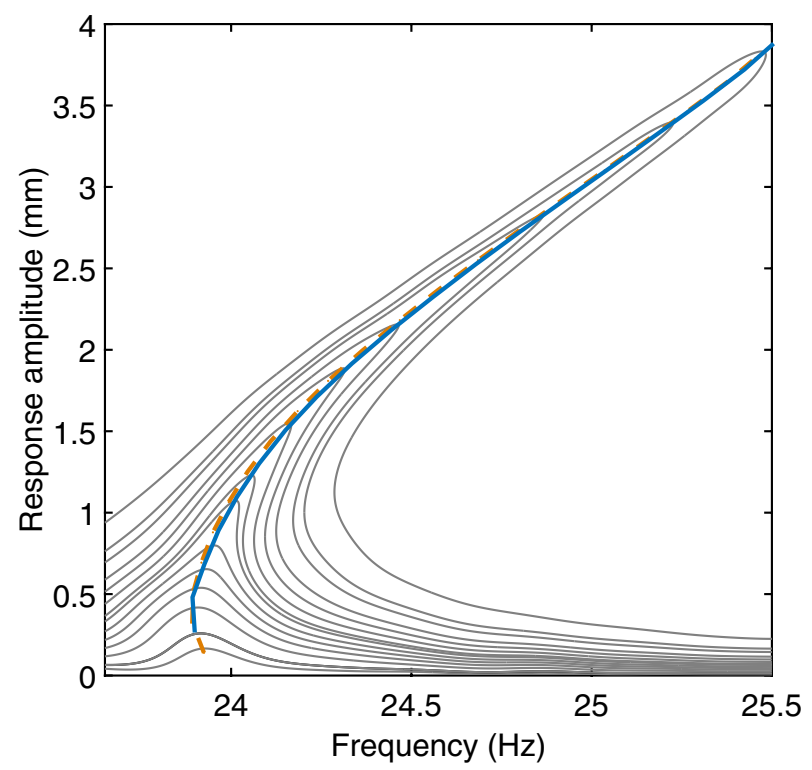

Fig. 6. Two backbone curves measured experimentally using CBC (-, - - ). (-) NLFR curves calculated at several constant forcing amplitudes using the GP regression and numerical continuation. 


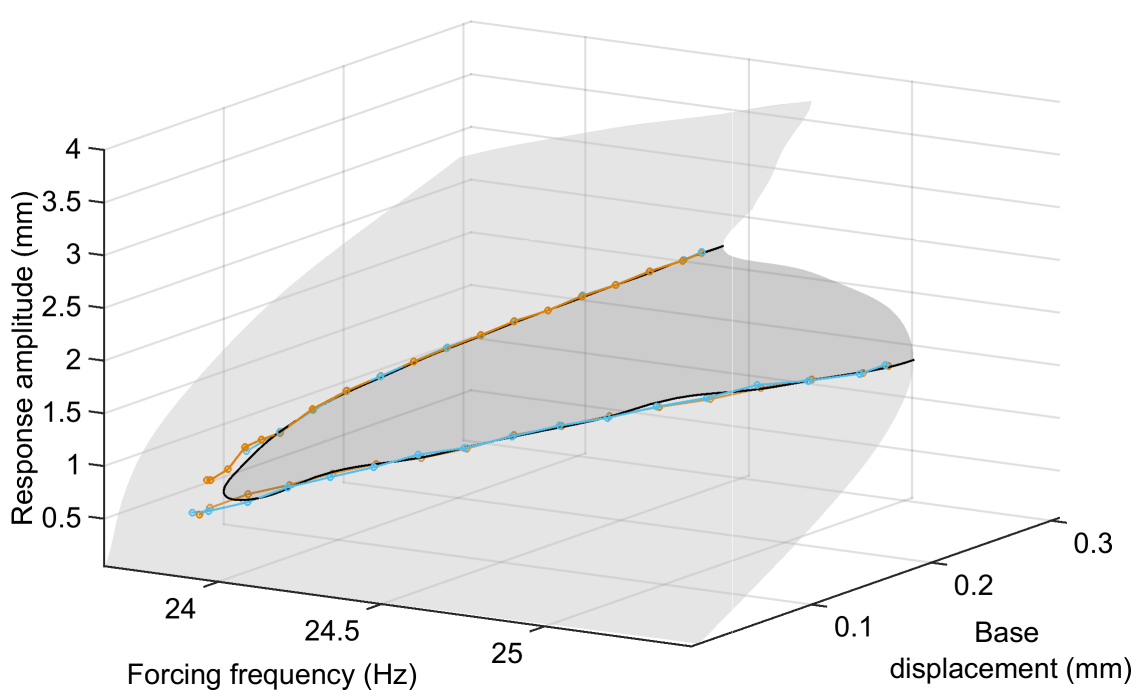

(a)

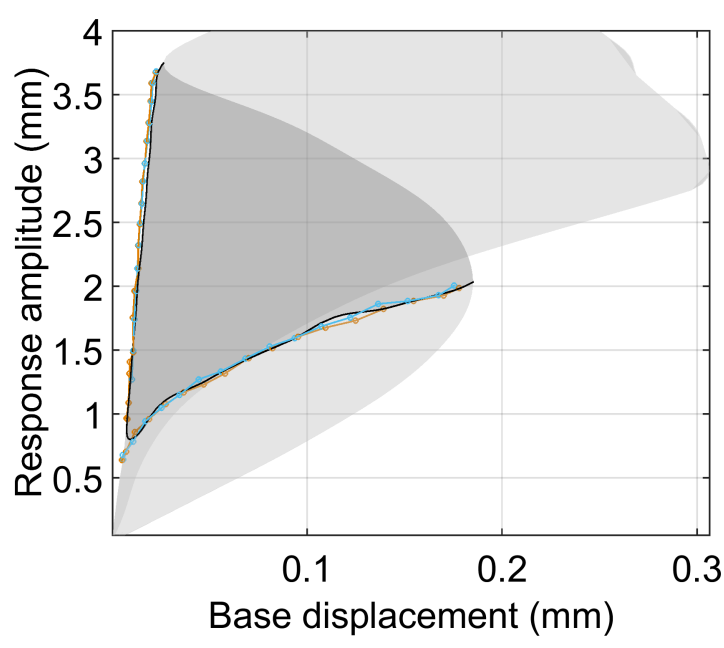

(b)

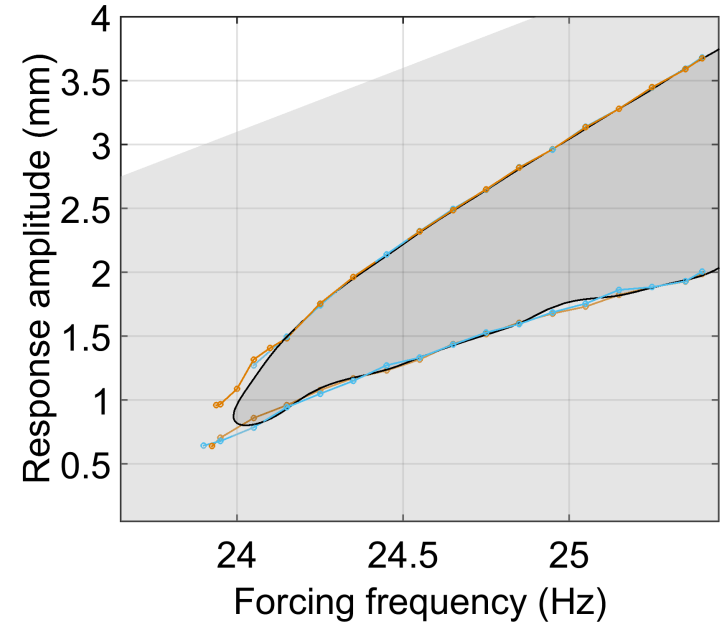

(c)

Fig. 7. $3 \mathrm{D}$ and 2D projections of the fold bifurcation curve with overlay of surface from Fig. 5 along with the GP calculated fold bifurcation curve (-). Regression based on $n=5(-)$ and $n=7(-)$ data points.

corresponding to the lower and upper parts of the bifurcation curve are obtained. Orange $(--)$ and blue $(-)$ curves correspond to data collected with $n=5$ and $n=7$ points per frequency, respectively.

Outside the region where the cusp bifurcation occurs, overall there is very good agreement between the LP curves extracted from the experiment and the one generated from the GP regression surface. Furthermore, the number of points used to estimate the position of the bifurcation in the experiment seems to have a limited influence on the results as the two curves, one based on using 5 and the other 7 points per domain, overlay almost perfectly. This result is confirmed in Fig. 8 where the bifurcation curves are compared in the forcingfrequency forcing-amplitude plane.

The curve obtained from the GP regression surface fluctuate slightly whereas the curves extracted directly from the experiment are smoother [see Figs. 7(a) and 7(c)]. These fluctuates are an artifact created by the regression and depend on the data and hyper-parameters selected. This issue is illustrated in Fig. 9 where the bifurcation curve from Fig. 5 is compared to another bifurcation curve obtained using GP regression on a second data set. This second data set corresponds to the first one complemented with six additional amplitude sweeps performed at $[23.65,23.75, \ldots, 24.15] \mathrm{Hz}$ around the 


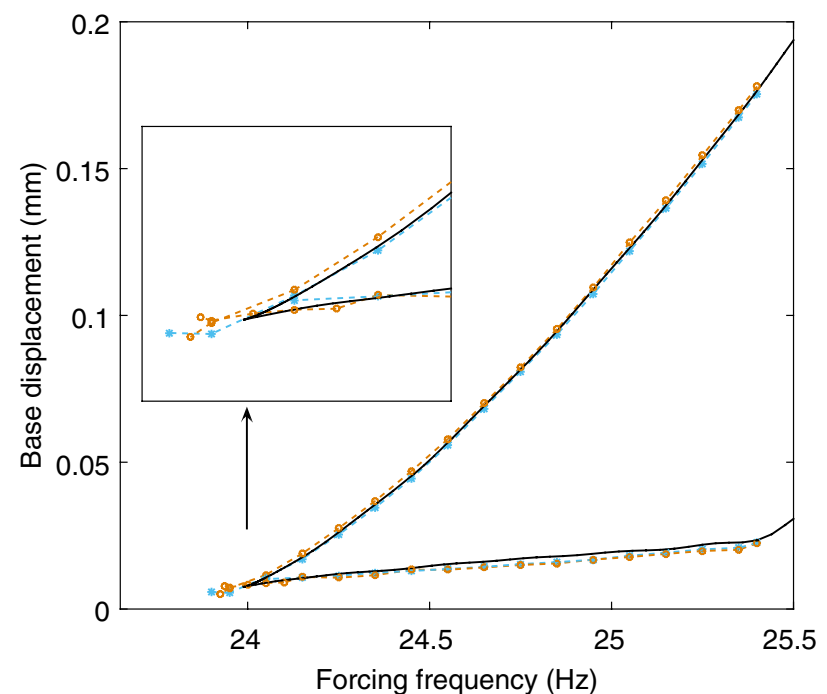

Fig. 8. Projection in parameter space of the fold bifurcation curve with overlay of the GP calculated fold bifurcation curve (-). Regression based on $n=5(-)$ and $n=7$ (-) data points.

cusp frequency. As shown in Fig. 9(a), the LP bifurcation curve fluctuates differently. Furthermore, Fig. 9(b) shows that the base displacement of the lower part of the bifurcation curve from the first data set is almost systematically underestimated. These variations in the GP results are attributed to a lack of data points and hence an excessive smoothing of the response surface in the bifurcation region. These results show that the discrepancies observed in Figs. 7 and 8 between the experimentally measured bifurcation curves and the one derived from the GP regression can be attributed, at least partially, to the GP regression. Interestingly, the position of the cusp bifurcation is almost identical for the two data sets.

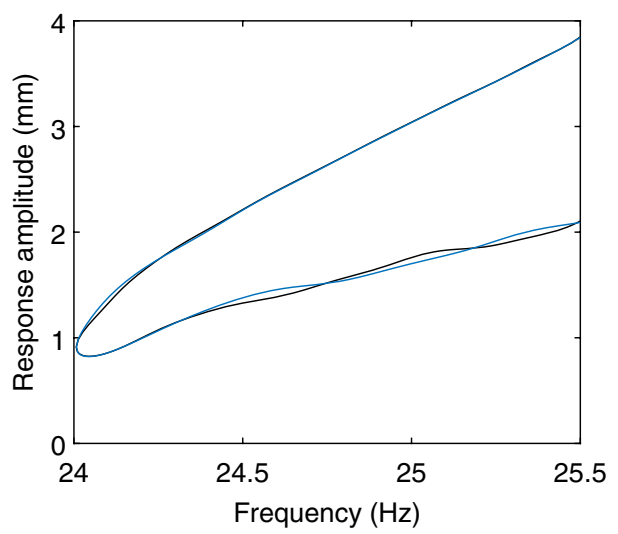

(a)
Discrepancies between measured and GPcalculated bifurcation curves increase around the cusp point. In this region, the curvature of the response surface decreases and the identification of the bifurcation point using experimental fold-point continuation becomes more sensitive to noise. Figures $10(\mathrm{a})-10$ (c) show this evolution through the data points collected at $24.25 \mathrm{~Hz}, 24.15 \mathrm{~Hz}$, and $24.05 \mathrm{~Hz}$, respectively. As in Fig. 3, data points are represented by $\bullet$, the cubic fit is in blue $(-)$, and the real root(s) of the polynomial first-order derivative are in red $(\diamond)$. The scale of the base-displacement axis is preserved between the three figures in order to highlight the flattening of the response surface as forcing frequency is decreased and the cusp bifurcation is approached.

Two LP bifurcations are detected in Fig. 10(c) which suggests that particular dynamics might be taking place for this specific frequency value. When two bifurcation points are detected, the algorithm collects additional data in a larger range of vibration amplitudes (cf. Sec. 2.3). Two bifurcation points are clearly visible in the new set of data points as shown in Fig. 10(d). The first bifurcation point is located just below $1.3 \mathrm{~mm}$, which is consistent with the value of $1.27 \mathrm{~mm}$ obtained in Fig. 10(c). The position of the second bifurcation is around at $0.9 \mathrm{~mm}$, which is significantly different from the $1.24 \mathrm{~mm}$ predicted in Fig. 10(c). From the previous results, it is clear that the data points in Fig. 10(c) do not cross the other LP bifurcation curve and that the prediction of a second LP is erroneous. However, the observation of two bifurcation points combined with the relative flattening of the surface fully justifies the collection of additional data.

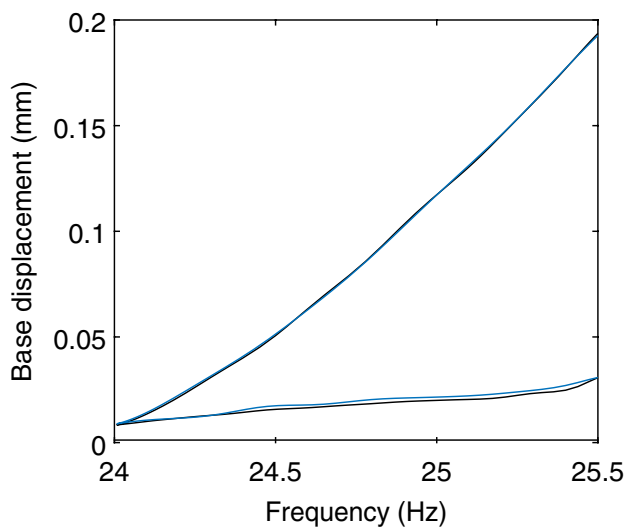

(b)

Fig. 9. GP calculated fold bifurcation curve for two different sets of data. 


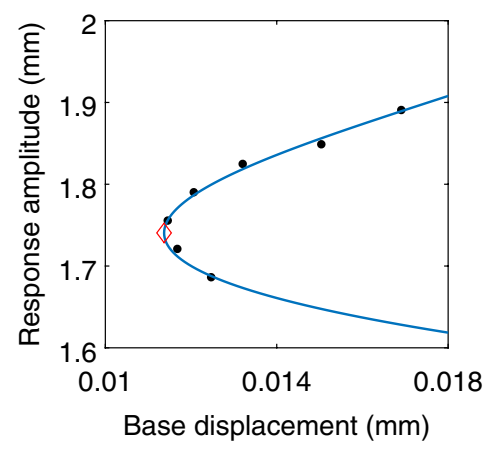

(a)

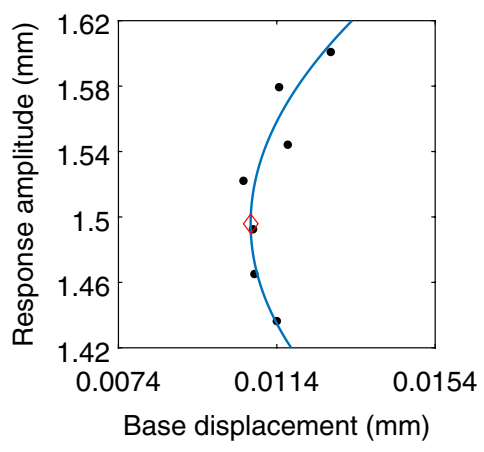

(b)

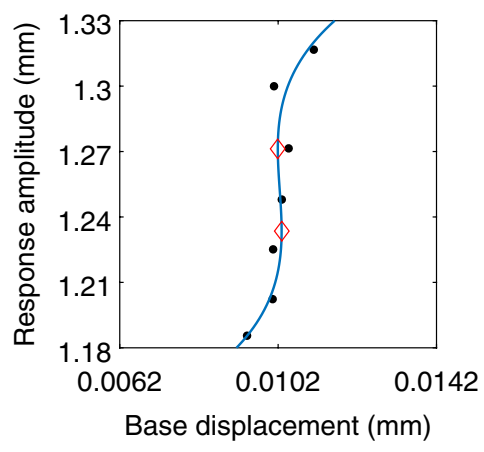

(c)

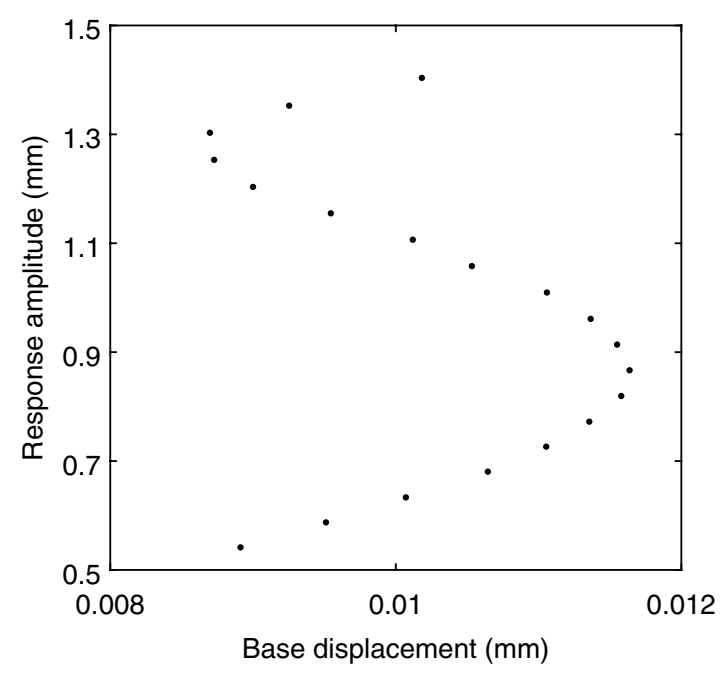

(d)

Fig. 10. (a)-(c) Data points collected at $24.25 \mathrm{~Hz}, 24.15 \mathrm{~Hz}$, and $24.05 \mathrm{~Hz}$ and (d) data points collected in a larger amplitude range at $24.05 \mathrm{~Hz}$. (•) Data point used for regression; (-) cubic regression; $(\diamond)$ estimated LP bifurcation point.

The interest in tracking directly the bifurcation curve in the experiment lies in the prospective reduction of the overall testing time. The largest number of data points collected to obtain both parts of the bifurcation curve was 215 , which is less than a third of the points collected for the S-curves. As the time necessary to perform the cubic regression is negligible compared to the time required to ensure the noninvasiveness of the controller and record the data, the overall testing time is also reduced by approximately a factor of 3 .

\section{Small-Gap Configuration}

The set-up is now investigated for the case where the distance between the mass and stator is $4.1 \mathrm{~mm}$. In this configuration, the dynamics of the system is more challenging than in Sec. 4 because the system exhibits a strong softening region. Oscillations are controlled using a PD feedback law whose proportional and derivative gains are 0.15 and 0.01 , respectively.

Figure 11 shows the S-curves collected between $16.5 \mathrm{~Hz}$ and $23.4 \mathrm{~Hz}$, with $0.1 \mathrm{~Hz}$ steps. The target coefficient $A_{1}^{*}$ was first incremented by $0.05 \mathrm{~mm}$ in order to overcome static friction in the system, followed by steps of $0.015 \mathrm{~mm}$. The performance of the controller was found to vary across the frequency range of interest such that the considered amplitude steps resulted in too large a variation of the response amplitude for frequencies greater than $22 \mathrm{~Hz}$. To preserve a sufficient number of points in the resonance region, finer steps of $0.01 \mathrm{~mm}$ were used for curves above $22 \mathrm{~Hz}$.

As in Sec. 4, GP regression is used to build a response surface from the collected S-curves (see Fig. 11). This surface is then analyzed to extract the curves of LP bifurcations (-) later used as 


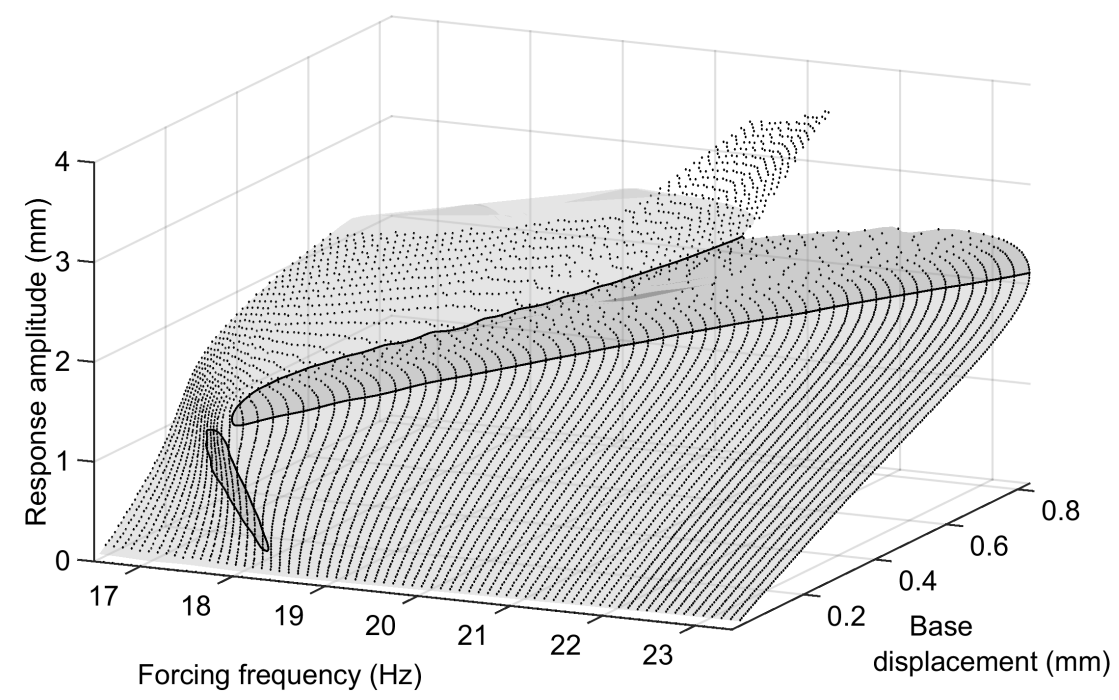

Fig. 11. Forced response of the SDOF shown in Fig. 4. (•) Amplitude of steady-state periodic responses measured during a series of amplitude sweeps. (Gray surface) The complete forced-response surface obtained using GP regression. (Dark-gray regions) Region where periodic responses are unstable without control. (-) Limit-point bifurcation curve obtained from the GP regression using numerical continuation.

a comparative for our direct algorithm. In the new configuration, the response surface presents a second region of unstable responses (dark-gray) between $17.4 \mathrm{~Hz}$ and $18.3 \mathrm{~Hz}$ which was not present for the previous configuration, see Fig. 5. This region, whose boundary is defined by a closed curve of LP bifurcations, is assumed to be due to the significant softening effect that is observed in this frequency range.

Figure 12(-, - - ) presents two backbone curves consecutively measured as described in Sec. 2.2. The curves overlay almost perfectly, which shows the consistency of the results and the excellent repeatability of the experiment. For response amplitudes smaller than $1.5 \mathrm{~mm}$, the backbone curve shows that the system resonance frequency decreases for increasing vibration amplitudes. Above $1.5 \mathrm{~mm}$, the resonance frequency increases at an almost constant rate.

A very good agreement between the backbone curves and the NLFR curves calculated using GP regression (-) is observed in the softening region and at the beginning of the hardening region [Fig. 12(a)]. However, for response amplitudes larger than $2.2 \mathrm{~mm}$, the NLFR curves present a number of distortions. In particular, the curves start to oscillate. One of the NLFR is split in two and presents a detached (isolated) response curve, while another does not fold inside the frequency range of interest. These distortions do not represent dynamical characteristics of the system and are artifacts created by the GP regression. Similar distortions are also visible on the curve of LP bifurcations in the resonance region [Fig. 12(b) $(-)]$. The LP curve exhibits dips above $19.5 \mathrm{~Hz}$ - precisely the frequency at which the NLFR starts to oscillate and splits in two. It is thought that the difficulty in capturing the resonance region is due to the shrinking of the basin of attraction that occurs in this region. This shrinking is known to affect the observability of stable responses in an experiment, leading sometimes to significant differences between experimental results and theoretical predictions where the system is stable in the sense of infinitesimal perturbations. This issue can be addressed using the concept of dynamical integrity [Thompson, 1989; Lenci et al., 2013]. Further tuning of the PD controller could also have been sufficient to improve the data captured in the resonance region but this was not considered in this study.

Figure 12(b) shows that both the softening and hardening parts of the backbone curve follow the bifurcation curves calculated using GP regression. In practice, this means it would be very difficult to measure the backbone curve without controlling the experiment. In this regard, the CBC method appears to be more robust than other existing methods such as resonant decay [Renson et al., 2016b].

Figure 13 presents, using 3D and different $2 \mathrm{D}$ projections, the LP bifurcation curves measured using Algorithm II. Starting from the bifurcations detected in the S-curve at $17.4 \mathrm{~Hz}$, the LP 


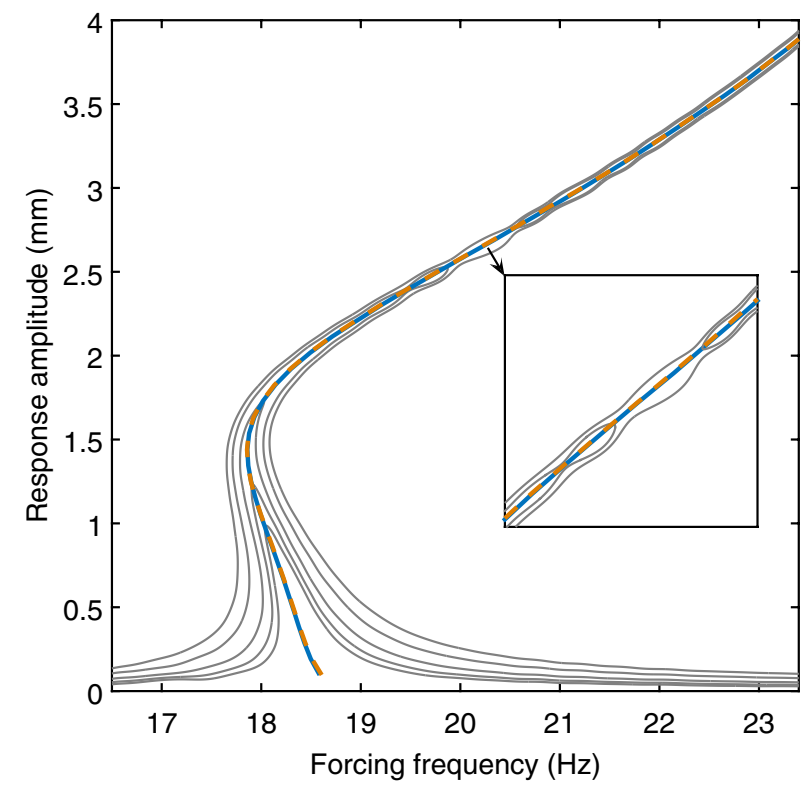

(a)

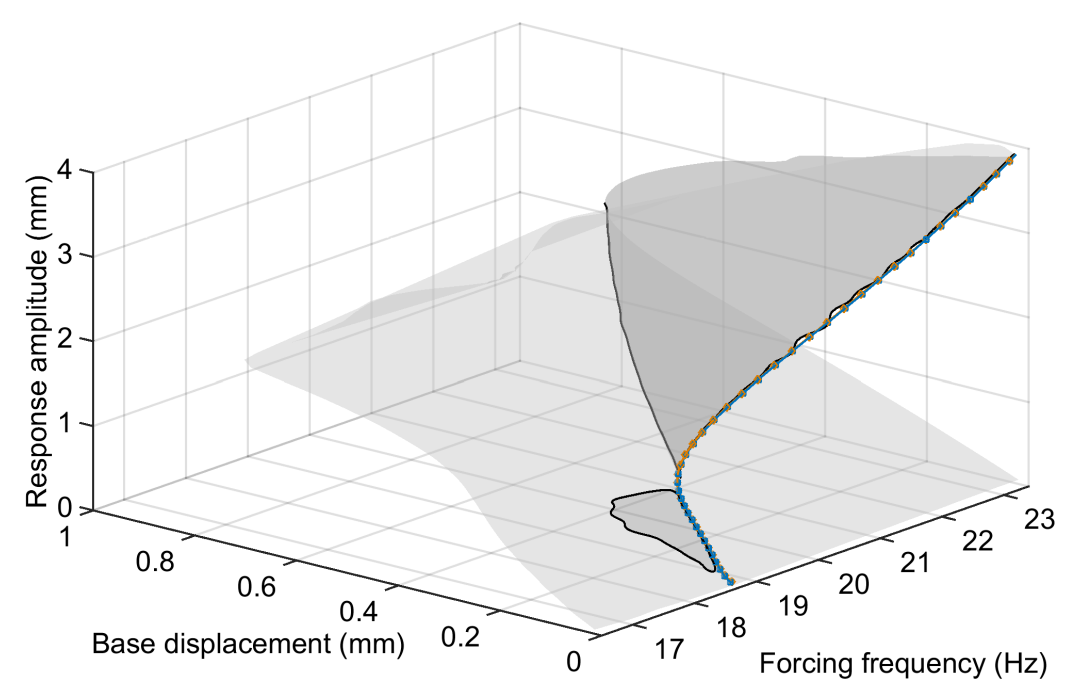

(b)

Fig. 12. Two backbone curves measured experimentally using CBC (-, - - ). (a) Comparison with NLFR curves (-) calculated at several constant forcing amplitudes using GP regression and numerical continuation and (b) comparison with GP calculated surface (gray) and LP bifurcation curves (-).

bifurcation curve in the softening region (-) was followed with frequency steps of $0.05 \mathrm{~Hz}$. Two parts, both ending at about $18.3 \mathrm{~Hz}$, were necessary to capture the curve. The portion of the curve closing the loop around $17.4 \mathrm{~Hz}$ could not be captured. In this region, the curve becomes almost vertical in frequency and cannot be captured unless very small frequency steps are taken [see Fig. 13(b)].

There is a good qualitative agreement between the measured and GP calculated bifurcation curves, though the base displacements from the experimental bifurcation tracking appear to be systematically underestimated [see Figs. 13(a)-13(c)]. Note that the noise in the base displacements measured with the laser combined to the relative flatness of the response surface in the softening region prevented us from having a robust identification of the bifurcation point. This issue was addressed by estimating the base displacement with the accelerometer signal. 


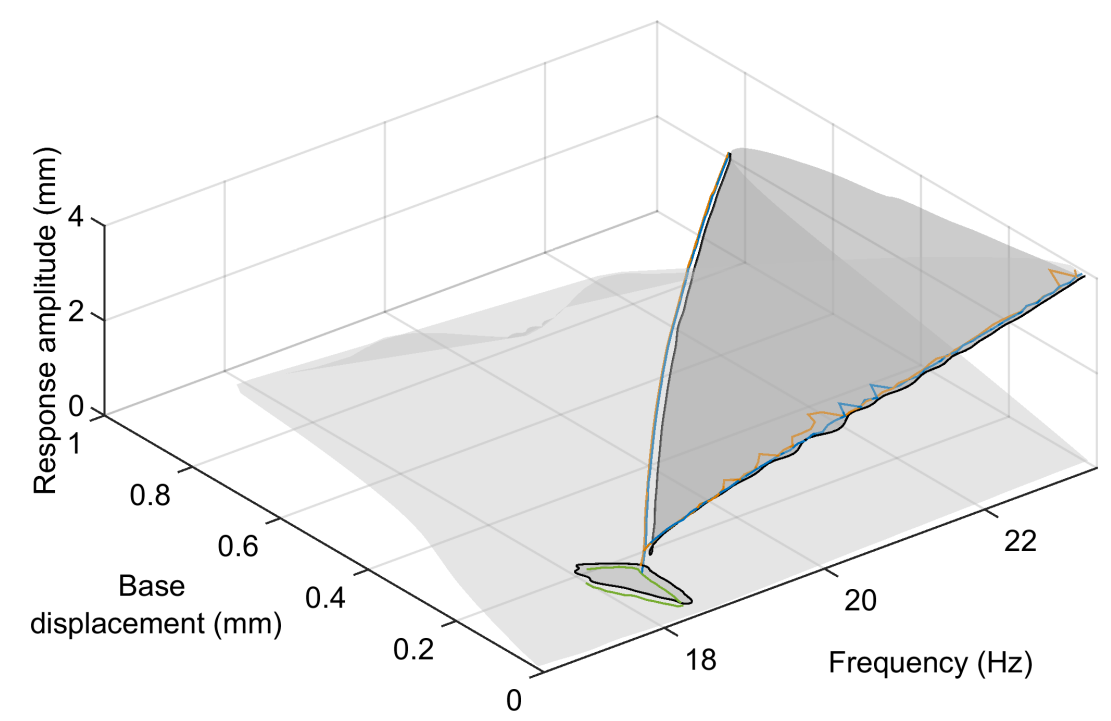

(a)

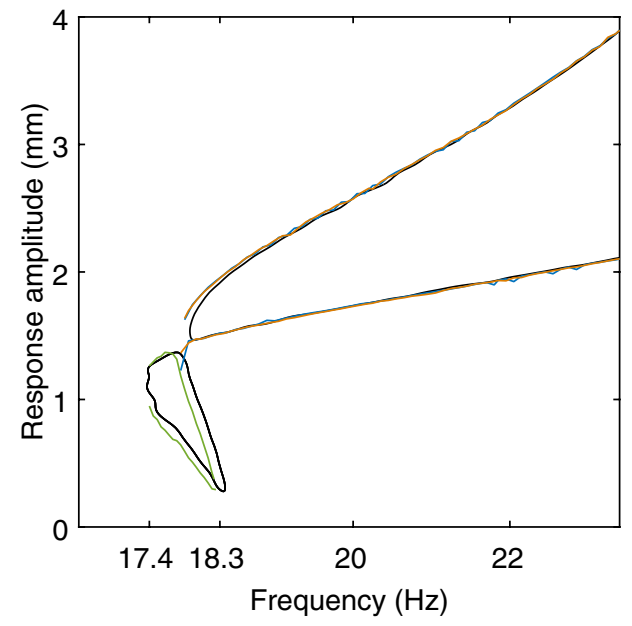

(b)

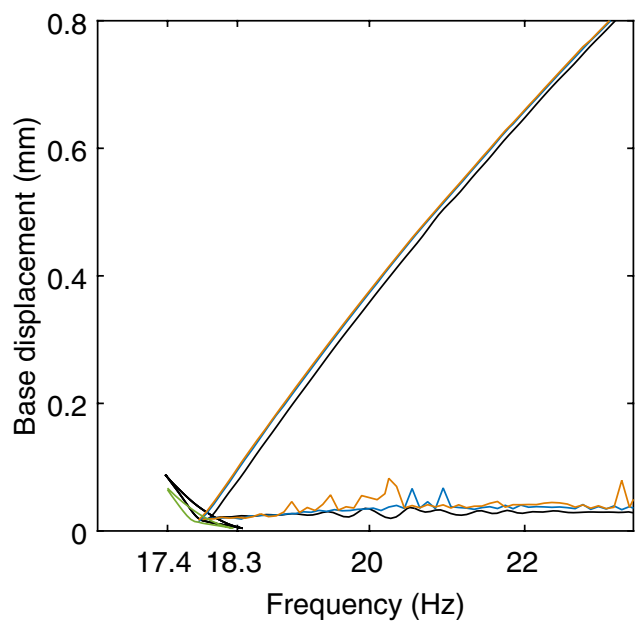

(c)

Fig. 13. 3D and 2D projections of the fold bifurcation curve with overlay of surface from Fig. 5 along with the GP calculated fold bifurcation curve (-). Regression based on $n=5(-), n=7(-), n=9(-)$ data points.

In the hardening region, the LP curves were tracked using two different numbers of data points per frequency step $(n=5(-)$ and $n=7(-))$. The curves corresponding to the largest base displacements agree almost perfectly and match very well the GP calculated bifurcation curve, which shows the consistency and repeatability of the experiment and the proposed method. Contrary to the curves in the softening region, the measured base displacement appears systematically larger than the value obtained with GP regression. In the resonance region (i.e. where the base displacements are the smallest), the bifurcation curves overall agree well with the GP regression although they appear noisier.

In contrast to the findings with the previous, large-gap, configuration, here the algorithm could not always stop automatically after the cusp bifurcation due to the presence of the other bifurcation curves in the softening region. As shown in Fig. 13(b), this issue was more pronounced when tracking the low-amplitude curve. For both runs (with $n=5$ and $n=7$ ), the algorithm had to be terminated manually. For this configuration of the nonlinearity, directly tracking the bifurcation point is approximately five times faster than collecting the 
data necessary to generate the complete response surface in Fig. 11. Although the speed-up factor would be smaller if adaptive stepping was considered to generate the S-curves in Fig. 11, this clearly demonstrates the attractive aspect of the proposed method.

\section{Conclusions}

This paper has proposed a simple method based on control-based continuation to track limit-point bifurcation curves directly during experimental tests. The method was demonstrated on a singledegree-of-freedom oscillator for two different configurations of the nonlinearity. The results were shown to agree very well with reference bifurcation curves calculated from detailed data sets capturing the complete response surface and Gaussian process regression. Compared to this latter approach, the proposed method was shown to considerably reduce the overall testing time. Moreover, the new direct limit-point bifurcation tracking method avoids the artificial distortions that are observed in the limitpoint curves calculated from the Gaussian process regression. Finally, the backbone curves of the system were accurately tracked for both configurations of the nonlinearity, which further demonstrates the broad applicability of the method originally developed in [Renson et al., 2016b].

In comparison to other ways of obtaining these results, we have shown that $\mathrm{CBC}$ is highly versatile and reliable. In contrast, obtaining limit-point bifurcation curves without the use of a closed-loop controller is tedious and error-prone, due to the fact that the curve itself is a stability boundary that can only be approached from one direction. Similarly, obtaining the backbone curve through free decay data can be highly unreliable since the decay trajectory can "jump" from one backbone curve to another when the modes of the system are close [Hill, 2016]. While other methods such as phaselocked loops [Peter \& Leine, 2016] are far more reliable, they are not very versatile and can only be used to investigate particular behaviors.

\section{Data Statement}

All the experimental data used in this paper have been deposited into the University of Bristol Research Data Repository and is publicly available for download [Renson, 2016].

\section{Acknowledgments}

The project leading to this application has received funding from the European Union's Horizon 2020 research and innovation programme under the Marie Sklodowska-Curie grant agreement No. 703204. L. Renson was also partly funded by Engineering Nonlinearity EPSRC programme grant, S. A. Neild by EPSRC fellowship EP/K005375/1, D. A. W. Barton by EPSRC grant EP/K032738/1, which are gratefully acknowledged.

\section{References}

Barton, D. A. W. \& Burrow, S. G. [2010] "Numerical continuation in a physical experiment: Investigation of a nonlinear energy harvester," J. Comput. Nonlin. Dyn. 6, 011010.

Barton, D. A. W. \& Sieber, J. [2013] "Systematic experimental exploration of bifurcations with noninvasive control," Phys. Rev. E 87, 052916.

Barton, D. A. W. [2015] "Real-time control hardware/software based on the beaglebone black," http://github.com/ dawbarton/rtc.

Barton, D. A. W. [2016] "Control-based continuation: Bifurcation and stability analysis for physical experiments," Mech. Syst. Sig. Process. B 84, 54-64.

Bureau, E., Schilder, F., Ferreira Santos, I., Thomsen, J. J. \& Starke, J. [2013] "Experimental bifurcation analysis of an impact oscillator - Tuning a noninvasive control scheme," J. Sound Vibr. 332, 58835897.

Cammarano, A., Hill, T. L., Neild, S. A. \& Wagg, D. J. [2014] "Bifurcations of backbone curves for systems of coupled nonlinear two mass oscillator," Nonlin. Dyn. 77, 311-320.

Dankowicz, H. \& Schilder, F. [2013] Recipes for Continuation (Society for Industrial and Applied Mathematics, Philadelphia, PA).

Detroux, T., Habib, G., Masset, L. \& Kerschen, G. [2015a] "Performance, robustness and sensitivity analysis of the nonlinear tuned vibration absorber," Mech. Syst. Sign. Process. 60-61, 799-809.

Detroux, T., Renson, L., Masset, L. \& Kerschen, G. [2015b] "The harmonic balance method for bifurcation analysis of large-scale nonlinear mechanical systems," Comput. Meth. Appl. Mech. Engin. 296, 1838.

Dhooge, A., Govaerts, W. \& Kuznetsov, Y. A. [2003] "Matcont: A Matlab package for numerical bifurcation analysis of ODEs," ACM Trans. Math. Softw. 29, 141-164.

Dick, A. J., Balachandran, B., DeVoe, D. L. \& Mote Jr., C. D. [2006] "Parametric identification of piezoelectric 
microscale resonators," J. Micromech. Microengin. 16, 1593.

Dimitriadis, G. \& Li, J. [2009] "Bifurcation behavior of airfoil undergoing stall flutter oscillations in low-speed wind tunnel," AIAA J. 47, 2577-2596.

Doedel, E. J., Paffenroth, R. C., Champneys, A. R., Fairgrieve, T. F., Kuznetsov, Y. A., Oldeman, B. E., Sandstede, B. \& Wang, X. J. [2000] "Auto2000: Continuation and bifurcation software for ordinary differential equations," Available at http://indy.cs. concordia.ca/auto/.

Doedel, E. J., Paffenroth, R. C., Keller, H. B., Dichmann, D. J., Galn-Vioque, J. \& Vanderbauwhede, A. [2003] "Computation of periodic solutions of conservative systems with application to the 3-body problem," Int. J. Bifurcation and Chaos 13, 13531381.

Draelants, D., Broeckhove, J., Beemster, G. T. S. \& Vanroose, W. [2013] "Numerical bifurcation analysis of the pattern formation in a cell based auxin transport model," J. Math. Biol. 67, 1279-1305.

Ewins, D. J. [2000] Modal Testing: Theory, Practice and Application, 2nd edition (Research Studies Press Ltd).

Gatti, G. [2016] "Uncovering inner detached resonance curves in coupled oscillators with nonlinearity," J. Sound Vibr. 372, 239-254.

Hill, T. L. [2016] "Modal interactions in nonlinear systems," PhD thesis, Department of Mechanical Engineering, University of Bristol.

Hill, T. L., Green, P. L., Cammarano, A. \& Neild, S. A. [2016] "Fast bayesian identification of a class of elastic weakly nonlinear systems using backbone curves," J. Sound Vibr. 360, 156-170.

Hodgkin, A. L. \& Huxley, A. F. [1952] "A quantitative description of membrane current and its application to conduction and excitation in nerve," J. Physiol. 117, 500-544.

Kerschen, G., Worden, K., Vakakis, A. F. \& Golinval, J. C. [2006] "Past, present and future of nonlinear system identification in structural dynamics," Mech. Syst. Sign. Process. 20, 505-592.

Kuether, R. J., Renson, L., Detroux, T., Grappasonni, C., Kerschen, G. \& Allen, M. S. [2015] "Nonlinear normal modes, modal interactions and isolated resonance curves," J. Sound Vibr. 351, 299-310.

Kurt, M., Eriten, M., McFarland, D. M., Bergman, L. A. \& Vakakis, A. F. [2015] "Methodology for model updating of mechanical components with local nonlinearities," J. Sound Vibr. 357, 331-348.

Kuznetsov, Y. A. [2004] Elements of Applied Bifurcation Theory, Applied Mathematical Sciences, Vol. 112 (Springer, NY).

Lenci, S., Rega, G. \& Ruzziconi, L. [2013] "The dynamical integrity concept for interpreting/predicting experimental behaviour: From macro- to nanomechanics," Philos. Trans. Roy. Soc. A: Math. Phys. Engin. Sci. 371.

Lugner, P., Pacejka, H. \& Plochl, M. [2005] "Recent advances in tyre models and testing procedures," Vehicle Syst. Dyn. 43, 413-426.

Peeters, B., Climent, H., De Diego, R., De Alba, J., Ahlquist, J. R., Carreo, J. M., Hendricx, W., Rega, A., Garca, G., Deweer, J. \& Debille, J. [2008] "Modern solutions for ground vibration testing of large aircraft," Proc. Int. Modal Analysis Conf. (IMAC), Orlando, FL, USA.

Peeters, M., Kerschen, G. \& Golinval, J. C. [2011a] "Dynamic testing of nonlinear vibrating structures using nonlinear normal modes," J. Sound Vibr. 330, 486-509.

Peeters, M., Kerschen, G. \& Golinval, J. C. [2011b] "Modal testing of nonlinear vibrating structures based on nonlinear normal modes: Experimental demonstration," Mech. Syst. Sign. Process. 25, 12271247.

Peter, S., Grundler, A., Reuss, P., Gaul, L. \& Leine, R. I. [2015] "Towards finite element model updating based on nonlinear normal modes," Proc. Int. Modal Analysis Conf. (IMAC), Orlando, USA.

Peter, S. \& Leine, R. I. [2016] "Experimental nonlinear modal analysis using a phase-locked-loop," Proc. 6th Int. Conf. Nonlinear Vibrations, Localization and Energy Transfer, Liège, Belgium.

Rasmussen, C. E. \& Williams, C. [2006] Gaussian Processes for Machine Learning (MIT Press, Cambridge, Massachusetts).

Renson, L., Noël, J. P. \& Kerschen, G. [2015] "Complex dynamics of a nonlinear aerospace structure: Numerical continuation and normal modes," Nonlin. Dyn. 79, 1293-1309.

Renson, L. [2016] "Experimental data for Experimental tracking of limit-point bifurcations and backbone curves using control-based continuation," dx.doi. org/10.5523/bris.1pfqddbxy8ry9ln8j5c0cbeqxe.

Renson, L., Barton, D. \& Neild, S. [2016a] "Experimental analysis of a softening-hardening nonlinear oscillator using control-based continuation," Proc. Int. Modal Analysis Conf. (IMAC), Orlando, USA.

Renson, L., Gonzalez-Buelga, A., Barton, D. A. W. \& Neild, S. A. [2016b] "Robust identification of backbone curves using control-based continuation," J. Sound Vibr. 367, 145-158.

Rosenberg, R. M. [1960] "Normal modes of nonlinear dual-mode systems," J. Appl. Mech. 27, 263268.

Schilder, F., Bureau, E., Santos, I. F., Thomsen, J. J. \& Starke, J. [2015] "Experimental bifurcation analysis - Continuation for noise-contaminated zero problems," J. Sound Vibr. 358, 251-266. 
Schlomer, N., Avitabile, D. \& Vanroose, W. [2012] "Numerical bifurcation study of superconducting patterns on a square," SIAM J. Appl. Dyn. Syst. 11, 447-477.

Seber, G. A. F. \& Lee, A. J. [2003] Linear Regression Analysis, 2nd edition, Probability and Statistics (John Wiley \& Sons, Inc.).

Seydel, R. [2010] Practical Bifurcation and Stability Analysis, Interdisciplinary Applied Mathematics, Vol. 5, 3rd edition (Springer, NY).

Sharma, S., Coetzee, E. B., Lowenberg, M. H., Neild, S. A. \& Krauskopf, B. [2015] "Numerical continuation and bifurcation analysis in aircraft design: An industrial perspective," Philos. Trans. Roy. Soc. London A: Math. Phys. Engin. Sci. 373, 2051.

Sieber, J. \& Krauskopf, B. [2008] "Control based bifurcation analysis for experiments," Nonlin. Dyn. 51, 365377.

Sieber, J., Krauskopf, B., Wagg, D., Gonzalez-Buelga, A. \& Neild, S. [2010] "Control-based continuation of unstable periodic orbits," J. Comput. Nonlin. Dyn. 6 , 011005 .

Sracic, M. W., Yang, S. \& Allen, M. S. [2012] "Comparing measured and computed nonlinear frequency responses to calibrate nonlinear system models," Proc. Int. Modal Analysis Conf. (IMAC), Orlando, USA.

Thompson, J. M. T. [1989] "Chaotic phenomena triggering the escape from a potential well," Proc. Roy. Soc. London A: Math. Phys. Engin. Sci. 421, 195.

Thompson, J. M. T. [2015] "Advances in shell buckling: Theory and experiments," Int. J. Bifurcation and Chaos 25, 1530001-1-25.

Vakakis, A. F., Manevitch, L. I., Mlkhlin, Y. V., Pilipchuk, V. N. \& Zevin, A. A. [2008] Normal Modes and Localization in Nonlinear Systems (Wiley-VCH Verlag GmbH, Weinheim, Germany).

Wang, C. \& Picaut, J. [2013] Understanding Enso Physics - A Review (American Geophysical Union), pp. $21-48$.

Worden, K. \& Tomlinson, G. R. [2001] Nonlinearity in Structural Dynamics: Detection, Identification and Modelling (Institute of Physics Publishing, Bristol and Philadelphia).

Zapico-Valle, J. L., Garcia-Diguez, M. \& AlonsoCamblor, R. [2013] "Nonlinear modal identification of a steel frame," Engin. Struct. 56, 246-259. 